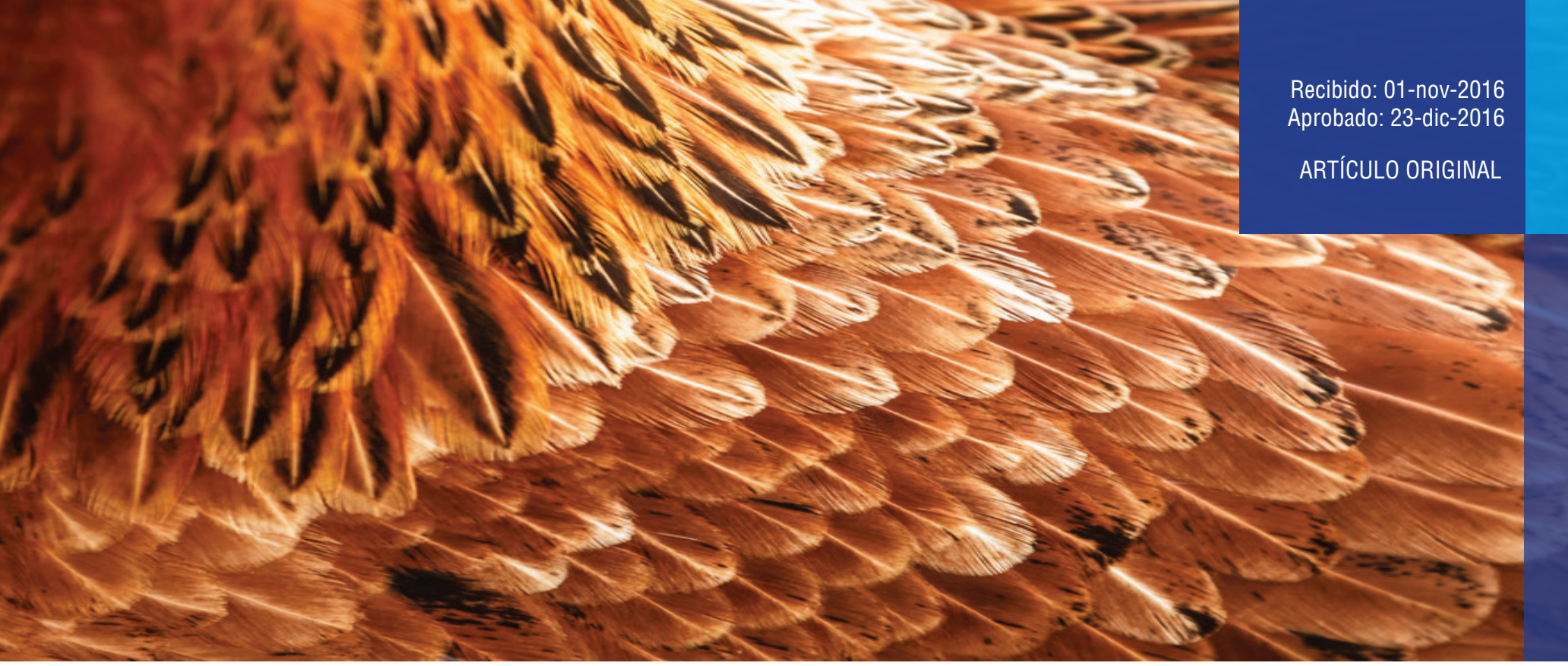

\title{
Obtención de termoplásticos a partir de plumas de pollo
}

\section{Thermoplastics obtaining from chicken feathers}
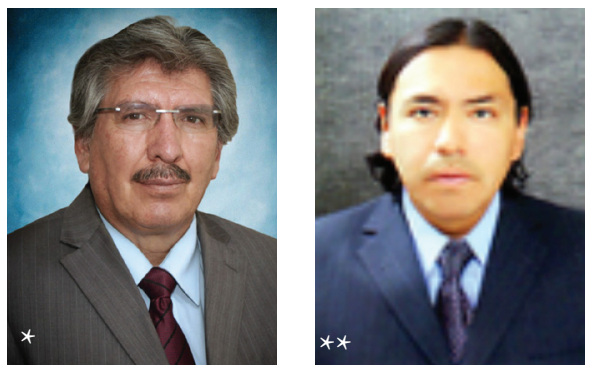

* Luis Calle Guadalupe, Ing. Químico, Magister en la Auditoría de Gestión de Calidad / Icalle@uce.edu.ec

**Washington Ruiz López, Ing. Químico, Magister en la Auditoría de Gestión de Calidad / wruiz@uce.edu.ec

Katherine Verduga

\section{Resumen}

Se obtuvieron películas de termoplásticos biodegradables a partir de queratina de plumas de pollo. Las plumas de pollo contienen en su mayor parte una proteína llamada queratina, la cual fue la sustancia principal de estudio en la investigación, ya que luego de ser extraída se procedió a polimerizar, para así producir el termoplástico. Se utilizó un método directo para obtener la Queratina, la misma que fue validada mediante pruebas en el FTIR (Fourier Transform Infrared Spectrometry), equipo que permitió separar los componentes de la misma, los cuales fueron confrontados con datos bibliográficos. La queratina fue sometida a un proceso de polimerización con glicerina y colágeno como agentes plastificantes y posteriormente se realizaron pruebas físicas, las cuales demostraron una mejor polimerización con colágeno que con glicerina; sin embargo, el polímero obtenido mostraba baja calidad. En conclusión, se comprobó que las concentraciones de los reactivos usados juegan un papel importante al momento de polimerización, ya que de estos dependen la calidad de la película biodegradable, así como también, el secado y el lavado de la queratina, procesos en los cuales se debe mantener un pH neutro o cercano.

Palabras clave: termoplásticos; queratina; polimerización; agente plastificante; películas biodegradables.

\section{Abstract}

Biodegradables thermoplastics were obtained from keratin of chicken feathers. The chicken feathers contain most of a protein called keratin, which was the main study in the investigation, since then to be obtained is proceeded to polymerize, to obtain the thermoplastic. It was used a direct method Keratin obtained that was validated by testing in the FTIR (Fourier Transform Infrared Spectrometry),

equipment which allowed to separate the components of the same, which were validated with bibliographic data. The keratin was subjected to a process of polymerization with glycerin and collagen as plasticizer agents, and was then carried out physical evidence, that showed a better polymerization with collagen that with glycerin; however, the polymer obtained showed low quality. In conclusion, it was found that the concentrations of the reagents used play an important role at the time of polymerization, since these depend on the quality of the film biodegradable, as well as the drying and washing of the keratin, processes in which there must maintain a neutral pH or nearby

Keywords: thermoplastics; keratin; polymerization; plasticizing agent; biodegradable films 


\section{Introducción}

El uso de productos plásticos es necesario en nuestra vida cotidiana, lo que ha generado un grave problema de contaminación ambiental a nivel mundial debido al tiempo que tarda en degradarse; además, aportan al calentamiento global ya que las fundas plásticas liberan gases de efecto invernadero (metano y dióxido de carbono principalmente) cuando se acumulan en los centros de acopio de desechos y comienzan a descomponerse ${ }^{1}$.

Los plásticos sintéticos tienen su origen en los derivados del petróleo, se estima que su degradación tarda alrededor de 500 años². Esta particularidad y la necesidad de su uso, hace indispensable investigar sobre la obtención de plásticos amigables con el ambiente, dando como resultado la creación de los biopolímeros.

Sonvarios los factores que demuestran la importancia de los biopolímeros: el alto costo de las resinas derivadas del petróleo, la creciente conciencia de los consumidores acerca de la necesidad de proteger al ambiente, la madurez tecnológica ya alcanzada en la generación de productos de alto desempeño con estas resinas derivadas del petróleo. Esto explica que en los últimos años los fabricantes de envases han lanzado al mercado productos de plásticos biodegradables fabricados a partir de recursos renovables.

Los bioplásticos, a diferencia de los plásticos convencionales, tienen su origen en productos vegetales o animales; en el proceso de su elaboración emiten entre 0,8 y 3,2 toneladas menos de dióxido de carbono por tonelada en comparación de los plásticos derivados del petróleo y el tiempo de degradación es menor, constituyendo así una alternativa para la sustitución de una gran variedad de plásticos ${ }^{3 .}$

Actualmente, se está trabajando en todo el mundo para encontrar materias primas alternativas que permitan abaratar los costos.

Desde 1970, la industria avícola ecuatoriana ha crecido de manera sostenida debido al crecimiento de la población de nuestro país y los cambios en su alimentación (en promedio, cada habitante consume $23 \mathrm{~kg}$ de carne de pollo al año). Actualmente esta industria constituye un sector importante de la economía nacional, debido a su relación con otros sectores económicos, consolidándose una gran cadena agroindustrial que se preocupa del problema de la emisión de grandes volúmenes de contaminantes orgánicos mal procesados y que se están desperdiciand0 4 . Solamente en la provincia de Pichincha existen 44 plantas avícolas, y si tomamos como referencia una planta que faene alrededor de 200 pollos diarios, tenemos que un pollo de 45 días de edad, pesa $2.4 \mathrm{~kg}$ y el $6.5 \%$ de su peso $(150 \mathrm{~g})$ corresponde a plumas; en promedio, de los 200 pollos se generan $30 \mathrm{~kg}$ de plumas al día y se desechan alrededor de $11.000 \mathrm{~kg}$ de plumas al año. Si esta cantidad de plumas se procesa como materia prima, de ella se obtendría alrededor de $9.000 \mathrm{~kg}$ de queratina, ya que el $80 \%$ de su peso corresponde a esta proteína ${ }^{5}$.

La utilización de estos residuos agrícolas para hacer bioplásticos, permite dar una segunda vida a un residuo y obtener un polímero biodegradable. De llegar a obtener resultados favorables, en un futuro se podría utilizar este desecho barato y abundante en la elaboración de varios productos, en lugar de que acabe incinerado o almacenado en los centros de acopio de basura.

\section{Teoría}

Las proteínas: Son biopolímeros formados por gran número de unidades estructurales repetitivas. Debido a su gran tamaño, cuando estas moléculas se dispersan en un disolvente adecuado, forman siempre dispersiones coloidales con características que las diferencian de las disoluciones de moléculas más pequeñas ${ }^{6}$.

\section{Características de las proteínas}

Las proteínas son largas cadenas de aminoácidos unidas por enlaces peptídicos entre el grupo carboxilo $(-\mathrm{COOH})$ y el grupo amino (-NH2) de residuos de aminoácido adyacentes. Por hidrólisis, las moléculas de proteína se dividen en numerosos compuestos relativamente simples, de masa molecular pequeña, que son las unidades fundamentales constituyentes de la macromolécula, estas unidades son los aminoácidos, de los cuales existen veinte especies diferentes y que se unen entre sí mediante enlaces peptídicos.

Cientos y miles de estos aminoácidos pueden participar en la formación de la gran molécula polimérica de una proteína?.

Queratina: Proteína que se presenta en forma de micro fibrillas, como si fuesen una maroma o cuerda. Las proteínas siempre están formadas por cadenas de aminoácidos que se enlazan entre sí formando fibrillas. Está muy extendida en la naturaleza, además de encontrarse en la piel, pelo y uñas, se encuentra en la lana, las plumas, pezuñas, cuernos, etc.

La queratina está compuesta básicamente por un aminoácido de alto contenido de azufre. Las queratinas duras contienen entre un 150 un $18 \%$ de azufre, mientras que las blandas sólo tienen entre un 2 y un 4\%.

Los protómeros de queratina se unen entre sí para formar dímeros. El dímero es el primer precursor de la gran molécula de la queratina en el que entran en juego varias proteínas. La unión no tiene lugar de cualquier manera, sino de una forma muy concreta: una subunidad ácida se unirá con una subunidad básica. De esta forma, la macromolécula acabará teniendo la misma proporción de subunidades básicas que de subunidades ácidas.

Posteriormente, dos dímeros se unirán entre sí para formar tetrámeros. Multitud de tetrámeros se unen entre sí, unos detrás de otros, dando lugar a los protofilamentos; varios p rotofilamentos se un en en tre sí, en grupos de unos cuatro protofilamentos formando una protofibrilla, a partir de estas protofibrillas b ien c ohesionadas s e f ormarán I as grandes moléculas de queratina, uniéndose muchas subunidades y torsionándose formando un trenzado.

Polímeros: Un polímero es una gran molécula constituida por la repetición de pequeñas unidades químicas simples. En algunos casos la repetición es lineal, de forma semejante a como una cadena la forman sus eslabones, en otros casos las cadenas son ramificadas 0 interconectadas formando retículos tridimensionales. La unidad repetitiva del polímero es usualmente equivalente 0 casi equivalente al monómero o material de partida del que se forma el polímero ${ }^{8}$.

Generalmente, las unidades químicas de las cuales está conformado un polímero son llamadas monómeros. 


\section{Clasificación de los Polímeros}

En función de la respuesta mecánica que tenga el polímero frente a temperaturas elevadas, pueden ser:

Termoplásticos: Son polímeros que, al calentarse a determinadas temperaturas (sobre la temperatura vítrea Tg), se convierten en fluidos, permitiendo su moldeabilidad en la forma deseada, que quedará preservada al enfriarse pues se endurecen debido a la formación de cristales. Constituyen el grupo más importante y de mayor uso comercial de polímeros sintéticos. Generalmente a estos materiales se les aplica calor y presión. Su estructura general puede ser lineal 0 ramificada y como característica general son blandos y dúctiles. Como ejemplos comunes de termoplásticos se encuentran los polietilenos, los polipropilenos, el policloruro de vinilo, las poliamidas, los policarbonatos, los poliuretanos.

Elastómero: Son polímeros que contienen cadenas con mucha libertad de movimiento molecular (flexibilidad).P resentan d obles e nlaces a lo largo de la cadena, pero también se pueden usar monómeros tridimensionales. Son insolubles, pero hinchables con solventes orgánicos. Los elastómeros se utilizan por sus excelentes propiedades elásticas, como ejemplo, se puede citar al caucho natural y sintético, el caucho nitrilo, el caucho estireno, entre otros.

Termoestables: Son polímeros que no pueden fluir p or e fecto de la temperatura para ser remoldeados. Están conformados por moléculas de cadena larga que están entrecruzadas en una organización tridimensional. Son moléculas de alto peso molecular con fuertes enlaces covalentes, tienden a ser resinas de mucha rigidez, y al someterlos a temperatura elevada, en vez de ablandarse, promueven la descomposición química del polímero (carbonización). A temperatura ambiente estos polímeros son duros y frágiles. Generalmente estos polímeros tienen mejores propiedades mecánicas, químicas, térmicas, y resistencia eléctrica de todos los polímeros termodependientes. Como ejemplos de polímeros termoestables se tienen a las resinas vinilésteres, resinas epoxi, resinas fenólicas, resinas urea-formaldehído, etc .

\section{Métodos}

\section{Se aplicó un método directo, que consiste de los siguientes procesos:}

\section{Lavado y secado de las plumas}

Las plumas se adicionan a un recipiente con suficiente a gua, I a agitación es manual y ocasional. Después de 6 horas de lavado se desecha el agua, usando una malla adecuada y se repite el proceso por dos veces más. Las plumas húmedas son secadas en una estufa a $50{ }^{\circ} \mathrm{C}$ por 48 horas.

\section{Molienda}

Se realizó la molienda de las plumas usando un molino ultra centrífugo con un tamiz de diámetro de partícula de $2 \mathrm{~mm}$. El molino presentó la facilidad de elegir el diámetro de partícula sin la necesidad de tamizarlo.

\section{Despigmentación}

Se utilizó una solución de hipoclorito de calcio al 3\%(v/v.), con una relación de plumas/solución de $\mathrm{Ca}(\mathrm{ClO})_{2}$ (p/v) de $1 / 7$ por 30 minutos a una temperatura de $18-22^{\circ} \mathrm{C}$.

\section{Desmineralización}

Se utilizó una solución de ácido clorhídrico $(\mathrm{HCl}) 1$ M. Se trabajó con una relación de plumas/HCl (p/p) de 1/3 por 24 horas a una temperatura de $18-22{ }^{\circ} \mathrm{C}$. Después se procedió a lavar las plumas para eliminar el exceso de ácido en la muestra, controlando que el agua de lavado tenga un pH de hasta 5.

\section{Desacetilación}

Se añadió una solución de hidróxido de sodio $(\mathrm{NaOH})$ a diferentes concentraciones con agitación constante. La relación de plumas / solución de $\mathrm{NaOH}(\mathrm{p} / \mathrm{p})$ fue de $1 / 7$, se trabajó por una hora y a una temperatura de $100{ }^{\circ} \mathrm{C}$ con un baño de glicerina, después se lavó la queratina obtenida con agua destilada hasta que el agua de lavado tenga un pH de 10-13.

\section{Formación de películas}

El método utilizado para la elaboración de las películas biodegradables fue el de evaporación de solvente, que es el que da los mejores resultados para obtener películas de acuerdo a trabajos realizados con otros biopolímeros en la Universidad Técnica de Ambato, así como en la Escuela Politécnica Nacional.

\section{Resultados}

Tabla 1

Rendimiento de queratina a partir de soluciones de diferente concentración de $\mathrm{NaOH}$

\begin{tabular}{|l|c|c|c|c|}
\hline $\begin{array}{c}\text { SOLUCIÓN } \\
\text { NaOH } \\
(\%)\end{array}$ & $\begin{array}{c}\text { M.I.P } \\
(\mathbf{g})\end{array}$ & $\begin{array}{c}\text { M.I.P.D. } \\
(\mathbf{g})\end{array}$ & M.Q.O(g) & $\begin{array}{c}\mathbf{R} \\
(\%)\end{array}$ \\
\hline $\mathrm{C} 1(40)$ & 50 & 34.4 & 5.8 & 11.6 \\
\hline $\mathrm{C} 2(45)$ & 50 & 35.0 & 5.6 & 11.2 \\
\hline $\mathrm{C} 3(50)$ & 50 & 38.7 & 6.2 & 12.4 \\
\hline $\mathrm{C} 4(60)$ & 50 & 36.6 & 5.9 & 11.8 \\
\hline $\mathrm{C} 5(70)$ & 50 & 33.3 & 5.4 & 10.8 \\
\hline
\end{tabular}

Fuente y elaboración: Autores

Tabla 2

Características de la solución de queratina obtenida

\begin{tabular}{|l|l|}
\hline CARACTERÍSTICAS & RESULTADOS \\
\hline Color & Amarillo claro \\
\hline Olor & Inodoro \\
\hline Transparencia & Transparente \\
\hline Densidad & $1,2 \mathrm{~g} / \mathrm{cm}^{3}$ \\
\hline Índice de refracción & 1,42 \\
\hline Viscosidad & $0,65 \mathrm{Cp}$ \\
\hline
\end{tabular}

Fuente y elaboración: Autores 
Tabla 3

Resultados de los ensayos físicos y mecánicos de las biopelículas

\begin{tabular}{|c|c|c|c|}
\hline MUESTRA & $\begin{array}{c}\text { ESPESOR } \\
(\mathbf{c m})\end{array}$ & $\begin{array}{c}\text { HUMEDAD } \\
\mathbf{( \% )}\end{array}$ & $\begin{array}{c}\text { RESISTENCIA A } \\
\text { LA ROTURA } \\
\mathbf{K g} / \mathbf{c m}^{\mathbf{2}} \mathbf{)}\end{array}$ \\
\hline $\mathrm{Cl}$ & 0,02 & 15.6 & 9.8 \\
\hline $\mathrm{C} 2$ & 0,02 & 15.8 & 9.5 \\
\hline $\mathrm{C} 3$ & 0,05 & 16.0 & 11.3 \\
\hline $\mathrm{C} 4$ & 0.03 & 16.7 & 10.9 \\
\hline $\mathrm{C} 5$ & 0.04 & 15.9 & 11.2 \\
\hline
\end{tabular}

Fuente y elaboración: Autores

\section{Discusión}

Para la obtención de la queratina se utilizó las barbillas y los raquis de plumas de aves de pollo de aproximadamente 60 días de edad; se aplicó un método modificado que utiliza las propiedades del $\mathrm{NaOH}$ para extraer la proteína, obteniéndose una solución cuyas características fueron comparadas con la queratina comercial que se encuentra en el mercado nacional. La polimerización se probó con dos agentes plastificantes como el colágeno y la glicerina que son los reactivos que se disponen en la Facultad de Ingeniería Química, pero se debería experimentar con otros reactivos que presentan superiores propiedades plastificantes para obtener excelentes resultados. Mejorando la calidad de la película, se la puede utilizar como materia prima para elaborar bolsas de plástico tipo camiseta, que son las que se usan en los supermercados. Además, se debe realizar un estudio de las propiedades biodegradables que presenta la película obtenida.

\section{Conclusión}

La película obtenida en este estudio es de baja calidad como se demuestra en la tabla de resultados. Para obtener una polimerización de mejor calidad es necesario experimentar con otros agentes plastificantes c omo e I a Icohol p olivinílico q ue tiene óptimas propiedades, en relación al colágeno y la glicerina para formar películas, como emulsionante y como adhesivo. El rendimiento en la obtención de queratina es del $12.4 \%$, el cual se puede mejorar incorporando un agente reductor al proceso de desacetilación que ayude a la extracción de la queratina. El espesor de la película se encuentra dentro del rango establecido en la norma NTE INEN 2290 y la resistencia a la rotura se puede mejorar incorporando un agente plastificante de mejores características.

\section{Conflicto de interés}

Los autores declaran NO tener ningún conflicto de interés.

\section{Referencias bibliográficas}

[1] Núñez, A. (2014) Obtención de una película de bioplástico a partir del colágeno de las patas de pollo.

[2] Núñez, A. (2014) Op. Cit.

[3] Núñez, A. Op. (2014) Cit.

[4] Osejos, P. (2009) Plan de manejo industrial para empresas.

[5] Osejos, P. (2009) Op. Cit.

[6] García, T. (2010) Las Proteínas.

[7] García, T. Op. Cit. 2010.

[8] Seymour, R. y Carraher, C. (1995) Introducción a la química de los polímeros.

[9] Seymour, R. Op. Cit. 1995.

\section{Anexos:}

\section{Lavado, Secado y Molienda. Desacetilación.}

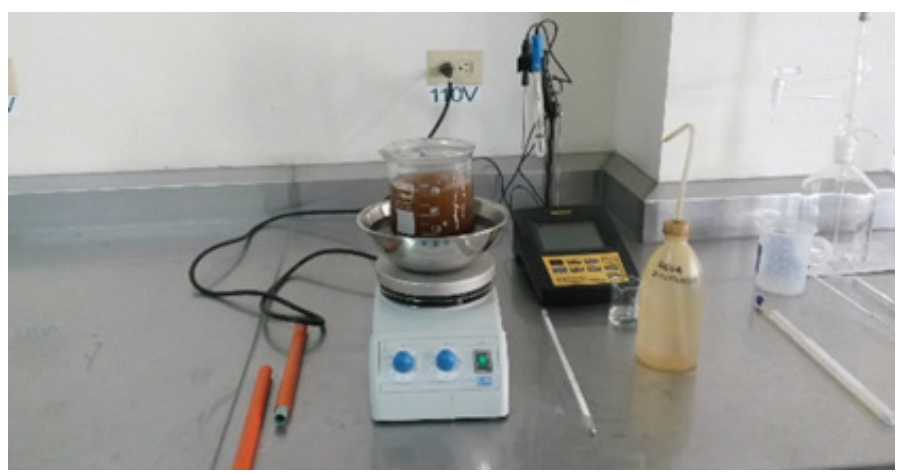

\section{Proceso de secado}

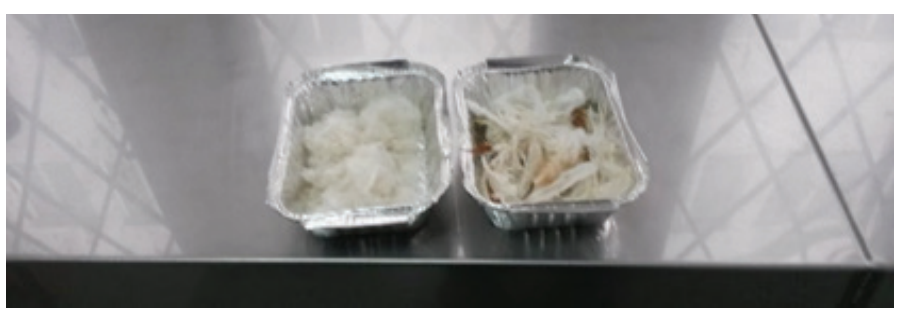

\section{Formación de Película}

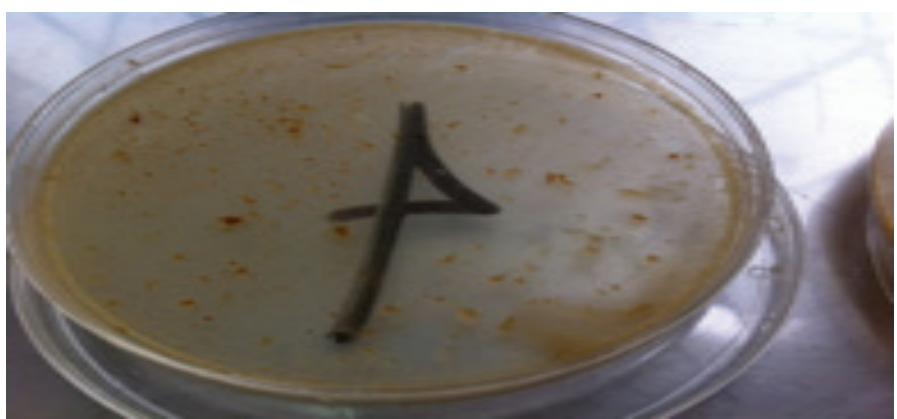

